Article

\title{
Classical Predictions for Intertwined Quantum Observables Are Contingent and Thus Inconclusive
}

\author{
Karl Svozil (D) \\ Institute for Theoretical Physics, Vienna University of Technology, Wiedner Hauptstrasse 8-10/136, \\ A-1040 Vienna, Austria; svozil@tuwien.ac.at
}

Received: 23 April 2020; Accepted: 8 May 2020; Published: 13 May 2020

\begin{abstract}
Classical evaluations of configurations of intertwined quantum contexts induce relations, such as true-implies-false and true-implies-true, but also nonseparability among the input and output terminals. When combined, these exploitable configurations (also known as gadgets) deliver the strongest form of classical value indefiniteness. However, the choice of the respective configuration among all such collections, and thus the relation of its terminals, remains arbitrary and cannot be motivated by some superselection principle inherent to quantum or classical physics.
\end{abstract}

Keywords: quantum mechanics; Gleason theorem; Kochen-Specker theorem; born rule; gadget graphs

PACS: 03.65.Ca; 02.50.-r; 02.10.-v; 03.65.Aa; 03.67.Ac; 03.65.Ud

\section{Quantum Clouds as Collections of Intertwined Contexts and Their Classical Doubles}

Quantum logic, as conceived of by von Neumann [1,2] and Birkhoff [3], is about the formal/theoretical universe of potential empirical observable propositions, as well as the algebraic relations and operations among them. Every single one of these observables is considered operational "subject to the experimenter's grace", as its actual measurement reflects the experimenter's (subjective) choice to indeed measure one of these potential observables, versus its (often continuity of) complementary ones (this choice is mostly supposed to be "ex machina"; that is, outside of the reach of quantum theory, and not subject to nesting [4-6]). Thereby, all the other, then counterfactual, observables remain in a "dormant/hypothetical" realm, an idealist $[7,8]$ "limbo" of sorts.

Even explorations allowing logical operations exclusively among simultaneously commeasurable observables [9,10] and permitting partial value definiteness [11,12] (in the recursion-theoretic sense of Kleene [13]) rely upon, and are thus valid relative to, such collections of counterfactuals. Thereby, the predictions/forecasts derived not for a single such collection of observables-here sometimes referred to as cloud or gadget-but for (finite) selections from the multitude of conceivable (finite) collections of observables may be inconsistent.

One way to conceptualize the (nonclassical) performance of quantized systems is in terms of (black) boxes with input and output terminals as interfaces [14]. Like zero-knowledge proofs [15] (a topic the late Specker became interested in), they are supposed to certify that they act "truly quantum mechanically" while at the same time not allowing any inspection (e.g., duplication or opening) other than their performance at the input-output terminals. Fulfillment/certification is usually obtained by the exhibition of certain features or signals usually not encountered by classical devices, among them complementarity and classical value indefiniteness (mostly pronounced as contextuality). For a great variety of such criteria, see Table 1 , as well as the references cited therein, later.

However, the signals obtained from these boxes are far from plain. Indeed, in what follows, we shall argue that, depending on which hypothetical configurations of (necessarily complementary) 
"intrinsic" observables are considered, any individual outcome can ad hoc be classically (re)interpreted as an indication of nonclassicality. Yet, the same outcome could also be in full conformity with a classical interpretation. A combination of such classical models allows any statistical prediction at the terminals. Moreover, there does not seem to exist any convincing reason to choose one of such configurations over another, thus giving rise to either contradictory or arbitrary ad hoc signal analysis.

Already, Specker [16] contemplated about generalized exotic behaviors even beyond quantum boxes, whereby his criteria for "weirdness" were inspired by scholastic counterfactuals (also known as Infuturabilien). The benign outcome of his fable was only made possible by the unmarried daughter's determined, alas futile, attempt to open the "wrong"-according to her father's strategy-box; at which point, he gave in, and marriage ensued. Quantum boxes and, as will be later argued, quantum clouds are not dissimilar: because of complementarity and classical value indefiniteness (also known as contextuality), complete knowledge of the situation is impossible by any known physical means. An immediate idealistic $[7,8,17]$ objection to the use of counterfactuals could be that the presupposition of the sort of omni-realism required for a classical analysis of quantum boxes cannot be operational [18] and supported by quantum mechanics. Indeed, the partial algebra approach of Kochen and Specker $[9,10,19]$ disallows operations among complementary observables whilst making heavy use of intertwined collections of complementary maximal operators (also known as contexts).

However, even classical models based on set representable partition logics [20] such as Moore's initial state identification problem [21] and also Wright's generalized urn model [22,23] mimic quantum complementarity to a certain degree-indeed, formally up to quantum logics with separable sets of two-valued states ([19], Theorem 0, p. 67). Thereby, nonseparability of quantum observables with respect to the set of two-valued states (interpretable as classical truth assignments) serves as a strict criterion for nonclassicality and also as a criterion against realizations by set-theoretical representable partition logics, even if such two-valued states exist.

In what follows, we shall further exploit counterfactual configurations of contexts that are intertwined (this terminology is borrowed from Gleason [24]) in one or more common observable(s). Such counterfactual configurations of contexts will be called clouds.

Graph theoretically ([25], Appendix), a context can be associated with a complete graph (also known as a clique). Its vertices are identified with the elements of the context. Adjacency is characterized by comeasurable exclusivity. Clouds are represented by collections of complete graphs (also known as cliques or contexts) intertwining at the respective vertices, thereby leaving the edges unchanged.

In quantum mechanics, contexts are identified with orthonormal bases, or equivalently with the maximal operators ([26], § 84, Theorem 1, p. 171), which can be (nonuniquely) formed by nondegenerate sums containing all the one-dimensional orthogonal projection operators associated with those respective bases. Elementary propositions are formalized by vectors of these bases of $d$-dimensional Hilbert space, or by the orthogonal projection operators associated with such vectors [3]. Graph theoretically, the vertices are represented by the basis vectors, and adjacency stands for the orthogonality of these vectors; that is, the edges represent the (pairwise) orthogonality relations between the vectors (vertices) (each vertex must be connected to all the other $d-1$ vertices in the respective context by an edge). Thereby, the graph representing a cloud has a faithful orthogonal representation $[27,28]$ in terms of the elements of the bases representing the respective contexts. The inverse problem of finding some faithful orthogonal representation of a given graph is still open. A necessary condition for the existence of intertwines is that the dimensionality of the vector space is higher than two because in fewer dimensions than three, contexts are either identical or disjoint.

Orthogonality hypergraphs [29] are compact representations of clouds that reveal their structural constituents by signifying contexts/cliques/bases: every complete graph $K_{d}$ is replaced by a single smooth curve (usually a straight line) containing distinguished points that represent the vertices. Thereby, the $d(d-1) / 2$ edges of any such complete graph $K_{d}$ are replaced with a single smooth curve. 
All the vertices "within" this smooth curve represent the mutually orthogonal vectors forming a $d$-dimensional basis.

Clouds may have various model realizations and representations: a particular cloud may have:

(i) a quantum mechanical realization in terms of intertwining orthonormal bases, as mentioned earlier;

(ii) a pseudo-classical realization in terms of partition logic, which in turn have automaton logic or generalized urn models;

(iii) a classical realization if there is only a single context involved;

(iv) none of the above (such as a tightly interlinked "triangle" configuration of three contexts with two vertices per context).

Suffice it to say that (i) does not imply (ii), and vice versa. Case (iii) can be interpreted as a subalgebra of all the other groups enumerated, as the cases (i) and (ii) are pastings of contexts or (Boolean) blocks [30].

\section{Enforcing Classical Two-Valued States}

The commonly used method for exploring nonclassicality is to consider configurations of type (i) with a quantum realization, upon which a classical interpretation, if it exists, is "enforced" in terms of uniform classical truth and falsity allocations of the associated propositions. Such value assignments can be formalized by two-valued states $s \in\{0,1\}$ or (classical truth) value assignments, which are additive and add up to one whenever the propositions are exclusive and within a single context.

The physical intuition behind this formalization is the observation that any $d$-dimensional context or maximal observable can be interpreted as an array of detectors after a $d$-port beam splitter [31]. In an ideal experiment, only one detector clicks (associated with the proposition that the system is in the respective state), whereas all the other $d-1$ detectors remain silent.

Such uniform classical interpretations are supposed to be context-independent; that is, the value on intertwining observables, which are common to two or more contexts, is independent of the context. Besides the context-independence of truth assignments at the intertwining observables, various variants of such measures assume conditions of increasing strength:

(I) The "measures" or value assignments employed in so-called "contextuality inequalities" merely assume that every proposition is either true or false, regardless of the other propositions in that context, which are simultaneously measurable [32]. This allows all possible $2^{d}$ possibilities of value assignments in a $d$-dimensional context with $d$ vertices, thereby vastly expanding the multitude of possible value assignments. With this expansion, all Kochen-Specker sets trivially allow value assignments.

(II) The prevalent assumption of two-valued states or value assignments, also used by Kochen and Specker [19], as well as Pitowsky [33], is that only a single one of the $d$ vertices within a $d$-dimensional context is true, and all the others are false; therefore, any isolated $d$-dimensional context can have only $d$ such standard two-valued value assignments.

(III) An even more restricted rule of value assignment abandons uniform definiteness and supposes [11,12,34] that, if all $d-1$ but one vertex in a $d$-dimensional context are false, the remaining one is true, and if one vertex within a $d$-dimensional context is true, all remaining $d-1$ vertices are false. These latter value assignments allow for partial functions, which can be undefined.

Type (III) implies Type (II), which in turn implies Type (I) value assignments. A set $S$ of two-valued states on a graph $G$ is $[35,36]$ :

(u) unital, if for every $x \in G$, there is a two-valued state $s \in S$ such that $s(x)=1$;

(s) separating, if for every distinct pair of vertices $x, y \in G$ with $x \neq y$, there is an $s \in S$ such that $s(x) \neq s(y)$ 
(f) full, if for every nonadjacent pair of vertices $x, y \in G$, there is an $s \in S$ such that $s(x)=s(y)=1$.

A full set of two-valued states is separating, and a separating set of two-valued states is unital. As will be detailed later TIFS/10-gadgets have a nonfull set, so two-valued states in the sense of (f). Nonseparability in the sense of (s) indicates nonclassicality. Nonunitality in the sense of (u) discredits the classical predictions of quantum clouds even to a greater degree, probably only challenged by a complete absence of two-valued states.

\section{Chromatic Separability}

As already discussed by Kochen and Specker ([19], Theorem 0), nonseparability of (at least one) a pair of nonadjacent vertices with respect to the set of two-valued states (interpretable as classical truth assignments) of a graph is arguably the most important signature of nonclassicality. It may be true even if there is an "abundance" of two-valued states. Nonseparability can also be expressed in terms of graph coloring.

A proper (vertex) coloring ([25], Appendix) of a graph is a function $c$ from the vertex set to a finite set of "colors" (the positive integers will do) such that, whenever $x$ and $y$ are adjacent vertices, $c(x) \neq c(y)$. The chromatic number of a graph is the least positive integer $t$ such that the graph has a coloring with $t$ colors.

A two-valued state on a (hyper)graph composed/pasted from contexts/cliques, all having an identical number of vertices/clique numbers $d$, can be obtained by "projecting/reducing" colors if the chromatic number of that graph equals $d$. In this case, in order to obtain a two-valued state, take any proper (vertex) coloring $c$ with $d$ colors and map $d-1$ colors into (the "new color") zero and one color into (the "new color") one. Just as the graph coloring $c$ itself, such mappings need not to be unique.

Note that the chromatic number of a complete graph must be equal to the clique number because Type (II) and (III) two-valued states require that every context/clique must have exactly one vertex with value assignment one (and zero for all the other vertices) (one might conjecture that the set of two-valued states induces graph colorings, in much the same way as it induces a partition logic [20]). At the same time, the clique number renders a bound from below on the chromatic number. Thus, if the chromatic number of a graph exceeds the clique number, no such two-valued states exist; the "phenomenological consequence" is that, for at least one context/clique, the color projection/reduction is constant-namely zero-on all vertices of that context/clique.

A coloring is chromatically separating two nonadjacent distinct vertices $x$ and $y$ in the vertex set of a graph if there exists a proper vertex coloring such that $c(x) \neq c(y)$. A set of colorings of a particular graph is said to be separating if, for any pair of distinct vertices, it contains at least one coloring, which separates those vertices. The separable chromatic number of a graph is the least positive integer $t$ such that the graph has a separating set of colorings with at most $t$ colors.

If the separable chromatic number is higher than the chromatic number of a given graph, then there exist nonadjacent vertices that cannot be "resolved" by any proper graph coloring, or, for that matter, by any derived projected/reduced two-valued state. As a consequence, the graph has no set-theoretic realization as a partition logic, although its chromatic number is the clique number, and there still may exist an abundance of proper colorings and two-valued states ([19], $\left.\Gamma_{3}, \mathrm{p} .70\right)$.

It would be interesting to translate (non)unitality and (non)fullness into (sets of) graph coloring. For reasons of brevity, we shall not discuss this here.

\section{Formation of Gadgets as Useful Subgraphs for the Construction of Clouds}

The commonly used method seeks cloud configurations with "exotic" classical interpretations. Again, exoticism may express itself in various forms or types. One way is in terms of violations on bounds on classical conditions of possible experience ([37], p. 229), such as Bell-type inequalities derivable from taking all (Type (II), and Type (I) for inequalities using only the assumption of noncontextuality [32]) value assignments, forming a correlation polytope by encoding those 
states into vertices, and solving the hull problem thereof [38-45]. Another, stronger [46] form of nonclassicality is the nonexistence of any such classical interpretation in terms of a Type (II) valued assignments $[16,19,24,33,47]$; or at least their nonseparability ([19], Theorem 0, p. 67).

The explicit construction of such exotic classical interpretations often proceeds by the (successive) application of exploitable subconfigurations of contexts; in graph theoretical terms, gadgets [48-50] defined as "useful subgraphs". Thereby, gadgets are formed from constituent lower order gadgets of ever-increasing size and functional performance (see also ([51], Chapter 12)):

1. Zeroth order gadget: a single context (also known as clique/block/Boolean (sub)algebra/ maximal observable/orthonormal basis). This can be perceived as the most elementary form of a true-implies-false (TIFS/10) [14]/01-(maybe better 10)-gadget [50,52] configuration, because a truth/value one assignment of one of the vertices implies falsity/value zero assignments of all the others;

2. First order "firefly" gadget: two contexts connected in a single intertwining vertex;

3. Second order gadget: two first order firefly gadgets connected in a single intertwining vertex;

4. Third order house/pentagon/pentagram gadget: one firefly and one second order gadget connected in two intertwining vertices to form a cyclic orthogonality hypergraph;

5. Fourth order 10-gadget: e.g., a Specker bug [14] consisting of two pentagon gadgets connected by an entire context; as well as extensions thereof to arbitrary angles for the terminal ("extreme") points $[12,50]$;

6. Fifth order true-implies-true (TITS) [14]/11-gadget [52]: e.g., Kochen and Specker's $\Gamma_{1}$ [19], consisting of one 10-gadget and one firefly gadget, connected at the respective terminal points (cf. Figure 6).

That is, gadgets are subconfigurations of clouds. Clouds can be interpreted as gadgets for the composition of bigger clouds.

For the sake of arguing for an idealistic $[7,8,17]$ and against a realistic usage of quantum clouds, configurations of intertwined contexts with two fixed propositions as "start" and "end" points $\mathbf{a}$ and $\mathbf{b}$ will be studied; as well as methods for constructing such configurations with particular relational properties. Whenever there is no preferred, less so unique, path connecting $\mathbf{a}$ and $\mathbf{b}$, all such connections should be treated on an equal basis. We shall call any such collection of counterfactual connections "clouds connecting $\mathbf{a}$ and $\mathbf{b}$ ", denoted by $C(\mathbf{a}, \mathbf{b})$, and depict it with a cloud shape symbol, as drawn in Figure 1 (this can in principle be generalized to more than two terminal points).

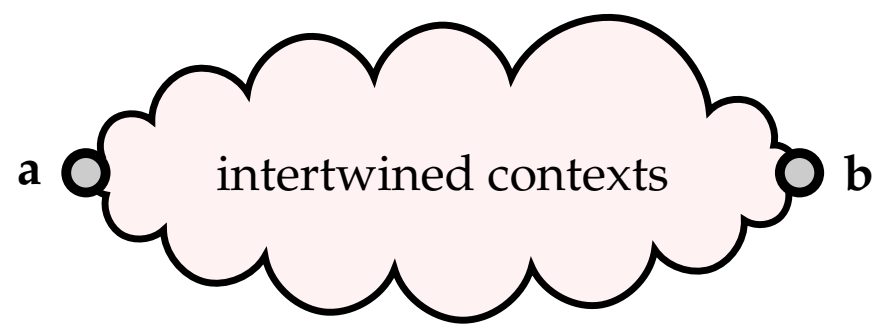

Figure 1. A collection of possible connections of counterfactuals organized in intertwining contexts and joining $\mathbf{a}$ and $\mathbf{b}$, depicted as a cloud $C(\mathbf{a}, \mathbf{b})$.

Thereby, as the endpoints $\mathbf{a}$ and $\mathbf{b}$ remain fixed, one can ask what kind of (classical) relational information can be inferred from such two-point quantum clouds. As it turns out, for fixed $\mathbf{a}$ and $\mathbf{b}$, quantum clouds can be found that realize a wide variety of conceivable relational properties between $\mathbf{a}$ and $\mathbf{b}$. Table 1 enumerates these relations. 
Table 1. Some (incomplete) history of the relational properties realizable by two-point quantum clouds.

\begin{tabular}{|c|c|c|}
\hline $\begin{array}{l}\text { If } A \text { is True } \\
\text { Classical Value Assignments }\end{array}$ & $\begin{array}{l}\text { Anecdotal, Historic } \\
\text { Quantum Realization }\end{array}$ & $\begin{array}{l}\text { Reference to Utility } \\
\text { or Relational Properties }\end{array}$ \\
\hline $\begin{array}{l}\text { imply } \mathbf{b} \text { is independent (arbitrary) } \\
\text { imply } \mathbf{b} \text { false (TIFS/10) } \\
\text { imply } \mathbf{b} \text { true (TITS) }\end{array}$ & $\begin{array}{l}\text { firefly logic } L_{12} \text { ([53], pp. 21, 22) } \\
\text { Specker bug logic ([9], Figure 1, p. 182) } \\
\text { extended Specker bug logic }\end{array}$ & $\begin{array}{l}\text { ([54], pp. 588-589), [14,55] } \\
\text { ([19], } \Gamma_{1}, \text { p. 68), } \\
\text { ([56], Sects. II, III, Figure 1), } \\
\text { ([57], Figure C.1. p. 67), } \\
\text { ([58], p. 394), [59-61], } \\
\text { [14,62-67] }\end{array}$ \\
\hline $\begin{array}{l}\text { iff } \mathbf{b} \text { true (nonseparability) } \\
\text { imply value indefiniteness of } \mathbf{b}\end{array}$ & $\begin{array}{l}\text { combo of intertwined Specker bugs } \\
\text { depending on Type (II), (III) assignments }\end{array}$ & $\begin{array}{l}\left([19], \Gamma_{3}, \text { p. } 70\right) \\
{[12,33]}\end{array}$ \\
\hline
\end{tabular}

\section{Quantum Clouds Enforcing Particular Features When Interpreted Classically}

For quantum mechanics, $\mathbf{a}$ and $\mathbf{b}$ can be formalized by the two one-dimensional projection operators $\mathbf{E}_{\mathbf{a}}=|\mathbf{a}\rangle\langle\mathbf{a}|$ and $\mathbf{E}_{\mathbf{b}}=|\mathbf{b}\rangle\langle\mathbf{b}|$, respectively. For the sake of demonstration, we shall study configurations in which $|\mathbf{a}\rangle=(1,0,0)$ and $|\mathbf{b}\rangle=\left(\frac{1}{\sqrt{2}}, \frac{1}{2}, \frac{1}{2}\right)$, that is the quantum prediction yields a probability $|\langle\mathbf{b} \mid \mathbf{a}\rangle|^{2}=\frac{1}{2}$ of finding the quantum in a state $|\mathbf{b}\rangle$ if it has been prepared in a state $|\mathbf{a}\rangle$. This configuration can be extended to endpoints with (noncollinear and nonorthogonal) arbitrary relative location by the techniques introduced in $[12,50]$.

(a) A quantum cloud configuration for which classical value assignments allow $\mathbf{b}$ to be either true or false if $\mathbf{a}$ is true is the firefly configuration ([53], pp. 21-22), depicted in Figure 2, with five classical value assignments of Type (II) [68].

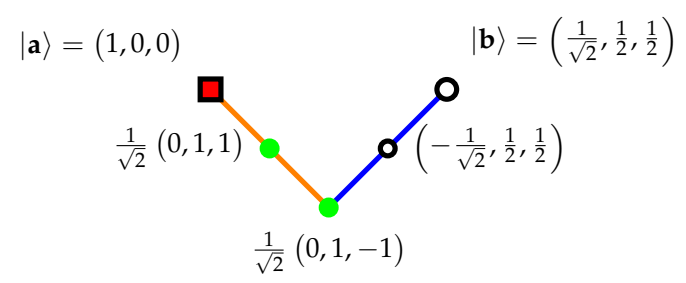

Figure 2. Orthogonality hypergraph of a cloud consisting of a firefly logic $L_{12}$ connecting a and b, such that, for Type (II) value assignments, a true-implies-b whatever (quantum 50:50). Truth is encoded by a filled red square, classical falsity by a filled green circle, and arbitrary truth values by circles (Type (III) value assignments are partial and thus undefined). $L_{12}$ consists of five vertices in just two intertwined blocks allowing a separating set of five two-valued states and therefore a set representable by partition logics.

(b) Already, Kochen and Specker utilized quantum clouds enforcing classical a true-implies-b false predictions and their compositions in the construction of a configuration that does not allow a uniform truth assignment (of Type (II)). Stairs ([54], pp. 588-589) has pointed out that the Specker bug ([9], Figure 1, p. 182) is a quantum cloud configuration that classically enforces a true-implies-b false: if a quantum system is prepared in such a way that $\mathbf{a}$ is true-that is, if it is in the state $\mathbf{E}_{\mathbf{a}}$-and measured along $\mathbf{E}_{\mathbf{b}}$, and $|\mathbf{a}\rangle$ and $|\mathbf{b}\rangle$ are not orthogonal or collinear, then any observation of $\mathbf{b}$ given $\mathbf{a}$ amounts to a probabilistic proof of nonclassicality: because although quantum probabilities do not vanish, classical value assignments predict that $\mathbf{b}$ never occurs. Minimal quantum cloud configurations for classical a true-implies-b false, as well as a true-implies-b true value assignments (of Type (II)) can be found in [14].

As Cabello has pointed out $[69,70]$, the original Specker bug configuration cannot go beyond the quantum prediction probability threshold $|\langle\mathbf{b} \mid \mathbf{a}\rangle|^{2}=3^{-2}$ because the angle between $\mathbf{a}$ and $\mathbf{b}$ cannot be smaller than $\arccos \frac{1}{3} \approx 1.23096$ radians $\left(71.5^{\circ}\right)$. A configuration ([71], Figure 5a) 
allowing Type (III) TIFS truth assignments with "maximally unbiased" quantum prediction probability $|\langle\mathbf{b} \mid \mathbf{a}\rangle|^{2}=\frac{1}{2}$ is a sublogic of a quantum logic whose realization was enumerated in [12], Table. 1, p. 102201-7. This is depicted in Figure 3. A proof of Theorem 2 in [50] contains an explicit parametrization of a single TIFS $/ 10$ cloud allowing the full range of angles $0<\angle \mathbf{a}, \mathbf{b}<\pi$.

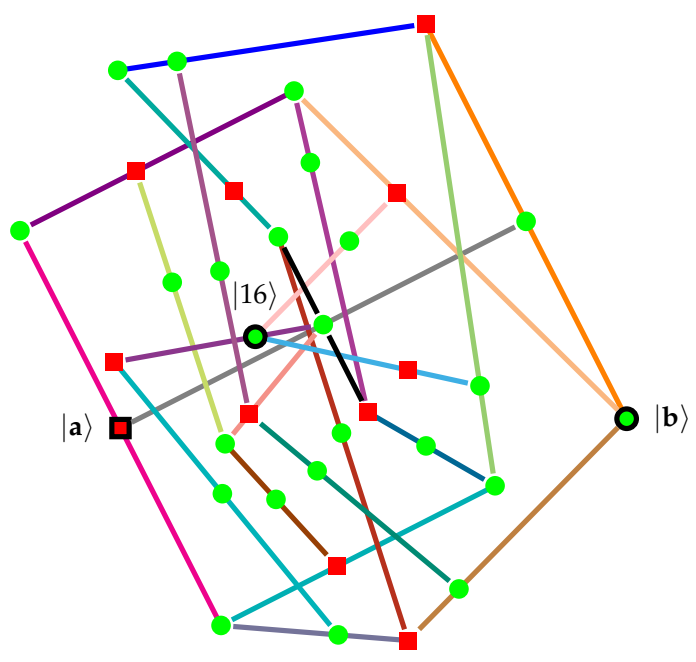

Figure 3. Orthogonality hypergraph of a nonfull/TIFS/10 cloud even for Type (III) value assignments. A faithful orthogonal realization was enumerated in [12], Table. 1, p. 102201-7. It consists of 38 vertices in 24 intertwined blocks, endowed with a nonseparating set of 13 two-valued states and therefore is not set representable by partition logics. The state depicted is the only one allowing a to be one. Moreover, this cloud has no unital set of two-valued states, as for all of them, the vertex represented by the vector $|16\rangle=\frac{1}{\sqrt{10}}(2 \sqrt{2}, 1,-1)$ and drawn as a solid black circle (and the associated observable) needs to be zero at all classical instantiations.

(c) Clifton (note added in the proof to Stairs ([54], pp. 588-589)) presented a a true-implies-b true (TITS) cloud ([56,72,73], Sects. II, III, Figure 1) inspired by Bell ([57], Figure C.1. p. 67) (cf. also Pitowsky ([58], p. 394)), as well as by the Specker bug logic ([56], Sects. IV, Figure 2). Hardy [59-61], as well as Cabello, among others $[50,63-67,69,70]$, utilized similar scenarios for the demonstration of nonclassicality ([74], Chapter 14). Figure 4 depicts an 11-gadget ([71], Figure 5b) with identical endpoints as the 10-gadget discussed earlier and depicted in Figure 3.

(d) Various parallel and serial compositions of 10- and 11-gadgets serve as a "gadget toolbox" to obtain clouds, which, if they are interpreted classically, exhibit other interesting relational properties. For instance, the parallel composition (pasting) of two quantum clouds of the 10-gadget type: one 10-gadget classically demanding a true-implies-b false and the other 10-gadget classically demanding $\mathbf{b}$ true-implies-a false, results in a quantum cloud that has two observables $\mathbf{a}$ and $\mathbf{b}$, which are classically always "opposite": if one is true, the other one is false, and vice versa.

(e) The parallel composition (pasting) of two quantum clouds of the TITS type, with one TITS, classically demanding a true-implies-b true and the other TITS classically demanding $\mathbf{b}$ true-implies-a true, results in a quantum cloud that has two observables $\mathbf{a}$ and $\mathbf{b}$, which are classically nonseparable, which is a sufficient criterion for nonclassicality ([19], Theorem 0, p. 67). As pointed out by Portillo [75], this is equivalent to $\mathbf{a}$ is true if and only if $\mathbf{b}$ is true (TIFFTS). Figure 5 depicts a historic example of such a construction. The serial composition of suitable TITS of the form $\mathbf{a}_{1}$ true-implies- $\mathbf{a}_{2} \cdots \mathbf{a}_{i-1}$ true-implies- $\mathbf{a}_{\mathbf{i}}$ true eventually yields two or more vectors $\mathbf{a}_{1}$ and $\mathbf{a}_{\mathbf{i}}$ that are mutually orthogonal; a technique employed by Kochen and Specker for the construction of a quantum cloud admitting no Type (II) truth assignment ([19], $\Gamma_{2}$, p. 69). 


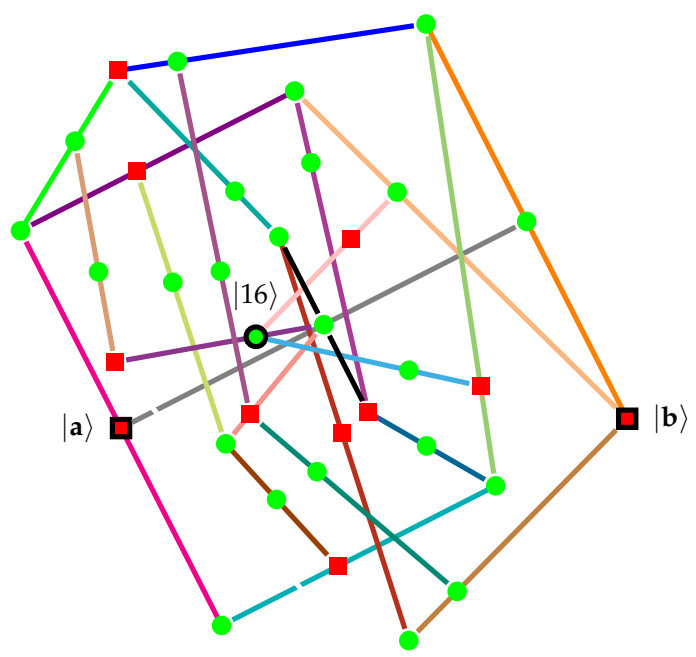

Figure 4. Orthogonality hypergraph of a TITS/11 cloud even for Type (III) value assignments. A faithful orthogonal realization was enumerated in [12], Table. 1, p. 102201-7. It consists of 38 vertices in 24 intertwined blocks, endowed with a nonseparating set of 13 two-valued states and therefore is not set representable by partition logics. The state depicted is the only one allowing a to be one. Moreover, this cloud has no unital set of two-valued states, as for all of them, the vertex represented by the vector $|16\rangle=\frac{1}{\sqrt{10}}(2 \sqrt{2}, 1,-1)$ and drawn as a solid black circle (and the associated observable) needs to be zero at all classical instantiations.

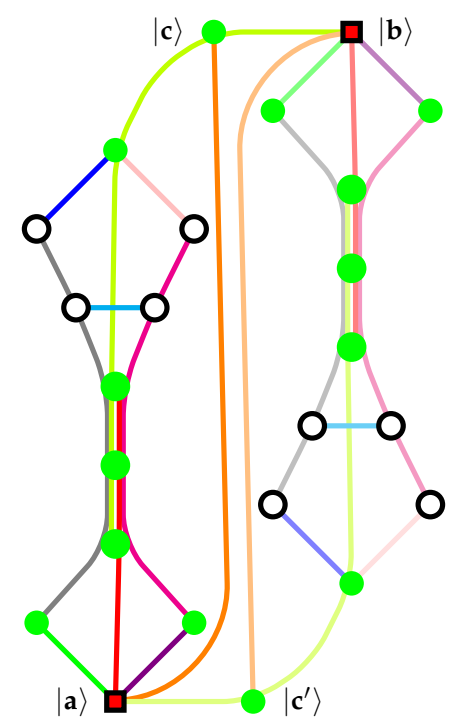

Figure 5. Orthogonality hypergraph of a TIFFTScloud for Type (II) value assignments, based on a minimal 11-gadget introduced in [14], Figure 6, for dimensions greater than two. In three dimensions, (i) the three orthogonal "middle" vertices intertwining four contexts vanish, (ii) the two vertices $|\mathbf{c}\rangle$ and $\left|\mathbf{c}^{\prime}\right\rangle$ coincide, and (iii) the two edges connecting $|\mathbf{c}\rangle$ with $|\mathbf{a}\rangle$ and $\left|\mathbf{c}^{\prime}\right\rangle$ with $|\mathbf{b}\rangle$ vanish, rendering the original Specker bug combo introduced by Kochen and Specker ([19], $\Gamma_{3}$, p. 70). Unlike the earlier configurations, this cloud does not allow 50:50 quantum probabilities. Because of the nonseparability of its set of two-valued states and its separable chromatic number higher than the clique number, it does not allow a set representation by partition logics.

(f) The parallel composition (pasting) of the two quantum clouds that respectively represent a 10-gadget and an 11-gadget and identical endpoints $\mathbf{a}$ and $\mathbf{b}$ yields $\mathbf{a} \mathbf{a}$ true-implies-b value indefinite cloud discussed in [12]. 


\section{Some Technical Issues of Gadget Construction}

The concatenation of intertwining gadgets needs to allow a proper faithful orthogonal representation of the resulting compound (hyper)graph, while at the same time preserving the structure of these gadgets. Thereby, the faithful orthogonal representations of the constituent gadgets cannot always be transferred easily to a faithful orthogonal representation of the resulting compound (hyper)graph.

Suppose, for the sake of a counterexample involving the duplicity of vertices after concatenations of gadgets, one would attempt to construct a $G((1,0,0),(0,1,1)) 11$ cloud (which would constitute a Kochen-Specker proof as the respective terminal points are orthogonal) by concatenating two 11-gadgets $G\left((1,0,0),\left(\frac{1}{\sqrt{2}}, \frac{1}{2}, \frac{1}{2}\right)\right)$ and $G\left(\left(\frac{1}{\sqrt{2}}, \frac{1}{2}, \frac{1}{2}\right),(0,1,1)\right)$ of the type depicted in Figure 4 by simply rotating all coordinates of the first gadget $\frac{\pi}{4}$ radians $\left(45^{\circ}\right)$ about the axis formed by $\mathbf{b}-\mathbf{a}$. Unfortunately, a straightforward calculation shows that these two 11-gadgets, with the faithful orthogonal realization taken from [12], Table 1, p. 102201-7, do not only have the vertex $\left(\frac{1}{\sqrt{2}}, \frac{1}{2}, \frac{1}{2}\right)$ in common as per the construction, but also the three additional vertices $\left(0, \frac{1}{\sqrt{2}}, \pm \frac{1}{\sqrt{2}}\right)$ and $(1,0,0)$.

Furthermore, gadgets may not be able to perform as desired. For instance, a standard construction in three dimensions, already used by Kochen and Specker ([19], Lemma 1, $\Gamma_{1}$, p. 68) for their construction of a 11-gadget $\Gamma_{1}$ from a Specker bug-type 10-gadget introduced earlier ([9], Figure 1, p. 182), is to take the terminal points $\mathbf{a}$ and $\mathbf{b}$ of some TIFS/10-cloud and form the normal vector $\mathbf{c}=\mathbf{a} \times \mathbf{b}$. In a second step, the vector:

$$
\begin{array}{r}
\mathbf{d}=\mathbf{b} \times \mathbf{c}=\mathbf{b} \times(\mathbf{a} \times \mathbf{b}) \\
=\mathbf{b}^{2} \mathbf{a}-(\mathbf{a} \cdot \mathbf{b}) \mathbf{b}=\mathbf{a}-(\mathbf{a} \cdot \mathbf{b}) \mathbf{b}=\mathbf{a}-\cos (\angle \mathbf{a}, \mathbf{b}) \mathbf{b}
\end{array}
$$

orthogonal to both $\mathbf{b}$ and $\mathbf{c}$ is formed. If $\mathbf{a}$ is true/one, then $\mathbf{b}$ (because of the 01-gadget), as well as $\mathbf{c}$ (because of orthogonality with a) must be false/zero. Therefore, $\mathbf{d}$ must be true, since it completes the context $\{\mathbf{b}, \mathbf{c}, \mathbf{d}\}$. The situation is depicted in Figure 6.

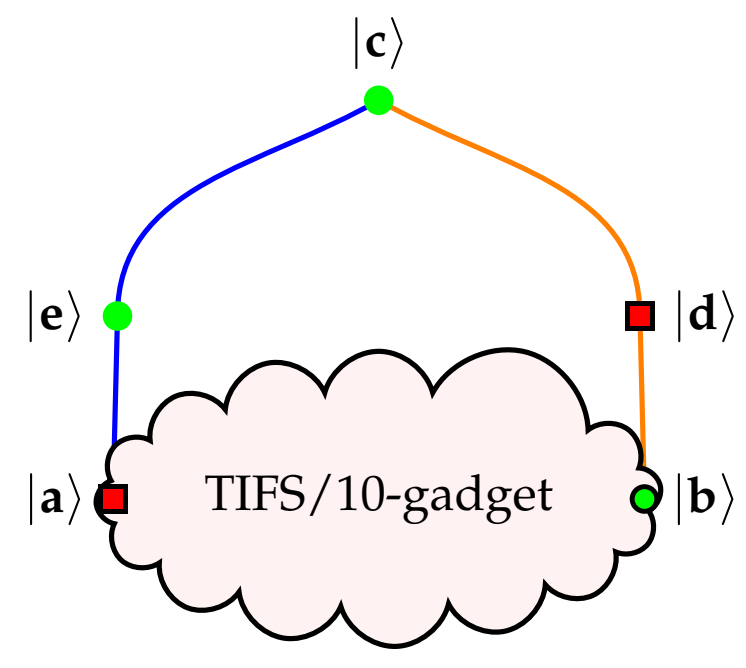

Figure 6. Standard construction used by Kochen and Specker [19, Lemma 1, $\Gamma_{1}$, p. 68]for obtaining an 11-cloud $C(\mathbf{a}, \mathbf{d})$ (or, because of symmetry, $C(\mathbf{b}, \mathbf{e})$ ) from a nonfull/TIFS/10-gadget $C(\mathbf{a}, \mathbf{b})$, involving two additional contexts $\{\mathbf{b}, \mathbf{d}, \mathbf{c}\}$ and $\{\mathbf{a}, \mathbf{c}, \mathbf{e}\}$.

If all goes well, the new cloud $C(\mathbf{a}, \mathbf{d})$ is of the TITS/11 type. This is not the case if one uses the TIFS/10-gadget depicted in Figure 3, as the vector $\mathbf{c}=\left(0,-\frac{1}{\sqrt{2}}, \frac{1}{\sqrt{2}}\right)$ and the new terminal vector $\mathbf{d}=\left(\frac{1}{\sqrt{2}},-\frac{1}{2},-\frac{1}{2}\right)$ also appear in the original TIFS/10-gadget. 
For very similar reasons (degeneracy or division through zero), the 10-gadget introduced in the proof of Theorem 3 in [50] and depicted in Figure 7 cannot be extended to an 11-cloud whose end terminals are the "maximal" angle $\frac{\pi}{4}$ radians $\left(45^{\circ}\right)$ apart. For all other allowed angles, an extension of this earlier construction of a TIFS/10-gadget to a TITS/11-cloud depicted in Figure 6 with (without loss of generality and for $\left.0<\angle \mathbf{a}, \mathbf{b} \leq \frac{\pi}{4}\right) \mathbf{a}=(1,0,0)$ and $\mathbf{b}=\frac{1}{\sqrt{1+x^{2}}}(x, 1,0)$ yields the new terminal vector of the TITS/10-cloud $\mathbf{d}=\frac{1}{\sqrt{1+x^{2}}}(1,-x, 0)=u_{20}$, which already occurs as the vector $u_{20}$ in the original TIFS/10-gadget. The only additional vertex $\mathbf{c}=(0,0,1)$ is from the edge connecting $u_{1}$ with $u_{3}$, as well as $u_{20}$ with $u_{22}$, thereby "completing" the two cliques/contexts $\left\{u_{1}, c, u_{3}\right\}$ and $\left\{u_{20}, c, u_{22}\right\}$. The angle between the two terminal points $u_{1}$ and $u_{20}$ of this TITS/11-gadget is $0<\arccos \frac{1}{\sqrt{1+x^{2}}} \leq \frac{\pi}{4}$ radians $\left(45^{\circ}\right)$ as $0<x \leq 1$. This configuration is also a TITS/10-cloud for the 17 pairs $u_{1}-\left\{u_{8}, u_{9}, u_{12}, u_{13}, u_{16}, u_{17}, u_{22}\right\}, u_{6}-u_{22}, u_{7}-\left\{u_{12}, u_{16}, u_{22}\right\}, u_{9}-u_{14}, u_{10}-u_{22}$, $u_{11}-\left\{u_{16}, u_{22}\right\}, u_{14}-u_{22}$, and $u_{15}-u_{22}$, respectively.

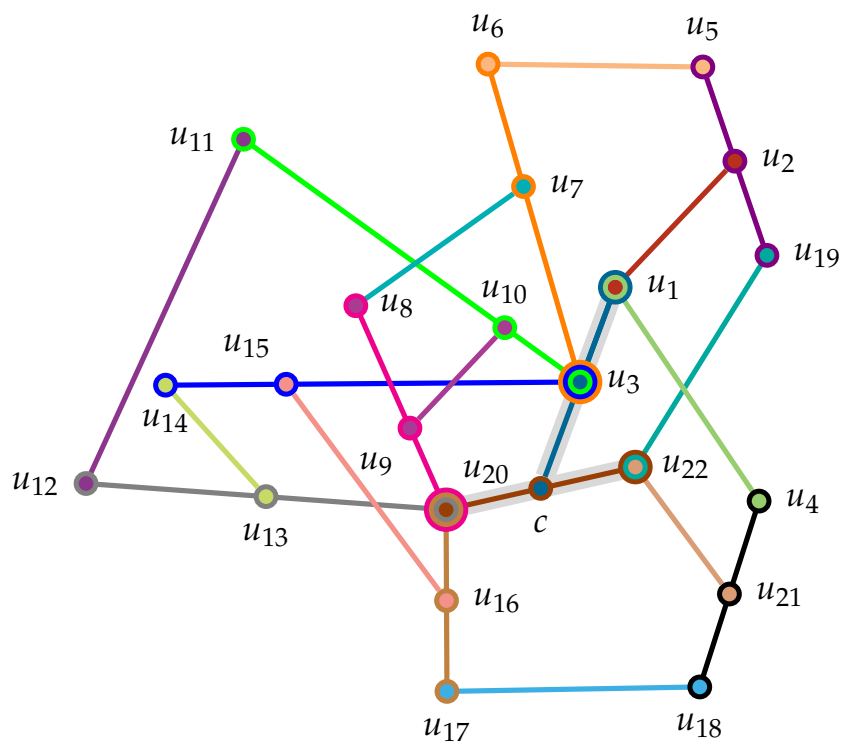

Figure 7. Orthogonality hypergraph from a proof of Theorem 3 in [50]. The advantage of this nonfull/TIFS/10-gadget is a straightforward parametric faithful orthogonal representation allowing angles $0<\angle u_{1}, u_{22} \leq \frac{\pi}{4}$ radians $\left(45^{\circ}\right)$ of, say, the terminal points $u_{1}$ and $u_{22}$. The corresponding logic including the completed set of 34 vertices in 21 blocks is set representable by partition logics because the supported 89 two-valued states are (color) separable. It is not too difficult to prove (by contradiction) that, say, if both $u_{1}$, as well as $u_{22}$ are assumed to be one, then $u_{2}, u_{3}, u_{4}$, as well as $u_{19}$, $u_{20}$, and $u_{21}$ should be zero. Therefore, $u_{5}$ and $u_{18}$ would need to be true. As a result, $u_{6}$ and $u_{17}$ would need to be false. Hence, $u_{7}$, as well as $u_{16}$ would be one, rendering $u_{8}$ and $u_{15}$ to be zero. This would imply $u_{9}$, as well as $u_{14}$ to be one, which in turn would demand $u_{10}$ and $u_{13}$ to be false. Therefore, $u_{11}$ and $u_{12}$ would have to be one which yields a complete contradiction even for Type (III) value assignments. It is also a TITS/11-gadget for the terminal points $u_{1}-u_{20}$, constructed by the standard construction depicted in Figure 6.

\section{Discussion}

It is important to notice that, for fixed terminal vertices, depending on the cloud chosen, very different classical predictions follow. Indeed, once the terminal vertices are fixed, it is not too difficult to enumerate a quantum cloud that, interpreted classically, predicts and demands any kind of input-output behavior. This renders an element of arbitrariness in the interpretation of quantum clouds.

The relevance of this observation lies in the conceivable interpretation of elementary empirical observations, such as a single particular click in a detector. Suppose a quantum is prepared in a pure 
state "along" a unit vector $\mathbf{a}$ and, when measured "along" $\mathbf{E}_{\mathbf{b}}=\mathbf{b}^{\dagger} \mathbf{b}$, "happens to activate a detector" corresponding to that state $\mathbf{b}$; that is, a detector associated with this latter property clicks. Depending on the quantum cloud considered, the following contradictory claims are justified:

1. if the quantum cloud allows both values, then the claim is that there is no determination of the outcome; the event "popped up" from nowhere, ex nihilo, or, theologically speaking, has come about by creatio continua (cf. Kelly James Clark's God-as-Curler metaphor [76]);

2. in the case of a 10-gadget, the system is truly quantum and cannot be classical;

3. in the case of an 11-gadget, the system could be classical;

4. in the case of a cloud inducing value indefiniteness, the claim can be justified that the system cannot be classical, as no such event (not even its absence) should be recorded. Indeed, relative to the assumptions made, the (non)occurrence of any event at all is in contradiction to the classical predictions.

Conversely, if the experimenter observes no click in a detector associated with the state $\mathbf{b}$, then, depending on the quantum cloud considered, the following contradictory claims are justified:

1. as mentioned earlier, if the quantum cloud allows both values, then there exists creatio continua (currently, this appears to be the orthodox majority position);

2. in the case of a 10-gadget, the system could be classical;

3. in the case of an 11-gadget, the system is truly quantum and cannot be classical;

4. just as mentioned earlier, in the case of a cloud inducing value indefiniteness, the claim can be justified that the system cannot be classical, as no such event (not even its absence) should be recorded.

As a result, depending on the quantum cloud considered, any (non)occurrence of some single outcome can be published (or rather marketed in venerable scientific journals) as a crucial experiment indicating that the associated system cannot be classical. Likewise, by taking other quantum clouds, any such outcome may be considered to be consistent with classicality: (non)classicality turns out to be means relative with respect to the quantum clouds considered. As quantum clouds are configurable for any input-output port setup, this is true for any measurement outcome.

The situation turns even more precarious if one considers quantum clouds with a nonunital (and nonseparable) set of two-valued states, such as the ones depicted in Figures 3 and 4: in the particular faithful orthogonal representation ([12], Table 1, p. 102201-7), the vector along $\frac{1}{\sqrt{10}}(2 \sqrt{2}, 1,-1)$ yields a classical prediction amounting to the nonoccurrence of the particular quantum observable. For another example, take a cloud introduced by Tkadlec ([36], Figure 2). It is based on a set of orthogonal vectors communicated to Specker by Schütte [77] and contains 36 vertices in 26 contexts/cliques, which allow six two-valued states enforcing eight vertices to be zero. In the particular faithful orthogonal representation of Tkadlec, those correspond to the vectors along $(1,0,0),(0,0,1),(1,0,1)$, $(1,0,-1),(2,0,-1),(1,0,2),(-1,0,2)$, and $(2,0,1)$. At the same time, the vector $(0,1,0)$ is forced to be one. Since without loss of generality, an orthogonal transformation can transform all of these vectors into other arbitrary directions (while maintaining the angles between vectors and, in particular, orthogonality), the assumption of such unital configurations and their classical interpretation immediately yields any desired contradiction with any individual measurement outcome.

This arbitrariness could be overcome by some sort of "superselection rule" prioritizing or selecting particular quantum clouds over other ones. However, in the absence of such superselection rules, a generalized Jayne's principle, or rather Laplace's principle of indifference, implies that any choice of a particular quantum cloud over other ones amounts to an "epistemic massaging" of empirical data and their nonoperational, misleading overinterpretation in terms of a speculative ontology $[7,8,17]$; or, to quote Peres [78], "unperformed experiments have no results". In contradistinction, it may not be too speculative to hold it for granted that the only operationally justified ontology is the assumption of a single one context or its associated maximal observable. 
Funding: The author acknowledges the support by the Austrian Science Fund (FWF): Project I 4579-N, and the Czech Science Foundation (GAČR): Project 20-09869L.

Acknowledgments: I kindly acknowledge enlightening discussions with Adan Cabello, José R. Portillo, and Mohammad Hadi Shekarriz. I am grateful to Josef Tkadlec for providing a Pascal program, which computes and analyses the set of two-valued states of collections of contexts. All misconceptions and errors are mine.

Conflicts of Interest: The author declares no conflict of interest.

\section{References}

1. von Neumann, J. Mathematische Grundlagen der Quantenmechanik, 2nd ed.; Springer: Berlin/Heidelberg, Germany, 1996. [CrossRef]

2. von Neumann, J. Mathematical Foundations of Quantum Mechanics; Princeton University Press: Princeton, NJ, USA, 1955.

3. Birkhoff, G.; von Neumann, J. The Logic of Quantum Mechanics. Ann. Math. 1936, 37, 823-843. [CrossRef]

4. Everett III, H. 'Relative State' Formulation of Quantum Mechanics. Rev. Mod. Phys. 1957, $29,454-462$. [CrossRef]

5. Wigner, E.P. Remarks on the mind-body question. In The Scientist Speculates; Good, I.J., Ed.; Heinemann and Basic Books: London, UK, 1961; New York, NY, USA, 1962; Springer: Berlin, Germany, 1995; pp. $284-302$. [CrossRef]

6. Everett, H, III. The Theory of the Universal Wave Function. In The Everett Interpretation of Quantum Mechanics: Collected Works 1955-1980 with Commentary; Barrett, J.A.; Byrne, P., Eds.; Princeton University Press: Princeton, NJ, USA, 2012; pp. 72-172.

7. Berkeley, G. A Treatise Concerning the Principles of Human Knowledge; Aaron Rhames for Jeremy Pepyat, Bookseller: Skinner-Row, Dublin, 1710.

8. Stace, W.T. The Refutation of Realism. Mind 1934, 43, 145-155. [CrossRef]

9. Kochen, S.; Specker, E.P. Logical Structures arising in quantum theory. In The Theory of Models: Proceedings of the 1963 International Symposium at Berkeley; North Holland: Amsterdam, The Netherlands; New York, NY, USA; Oxford, UK, 1965; pp. 177-189.

10. Kochen, S.; Specker, E.P. The calculus of partial propositional functions. In Proceedings of the 1964 International Congress for Logic, Methodology and Philosophy of Science, Jerusalem; North Holland: Amsterdam, The Netherlands 1965; pp. 45-57.

11. Abbott, A.A.; Calude, C.S.; Conder, J.; Svozil, K. Strong Kochen-Specker theorem and incomputability of quantum randomness. Phys. Rev. A 2012, 86, 062109. [CrossRef]

12. Abbott, A.A.; Calude, C.S.; Svozil, K. A variant of the Kochen-Specker theorem localising value indefiniteness. J. Math. Phys. 2015, 56, 102201. [CrossRef]

13. Kleene, S.C. General recursive functions of natural numbers. Math. Ann. 1936, 112, 727-742. [CrossRef]

14. Cabello, A.; Portillo, J.R.; Solís, A.; Svozil, K. Minimal true-implies-false and true-implies-true sets of propositions in noncontextual hidden-variable theories. Phys. Rev. A 2018, 98, 012106. [CrossRef]

15. Quisquater, J.J.; Quisquater, M.; Quisquater, M.; Quisquater, M.; Guillou, L.; Guillou, M.A.; Guillou, G.; Guillou, A.; Guillou, G.; Guillou, S. How to Explain Zero-Knowledge Protocols to Your Children. In Advances in Cryptology-CRYPTO' 89 Proceedings; Brassard, G., Ed.; Springer: New York, NY, USA, 1990; pp. 628-631. [CrossRef]

16. Specker, E. Die Logik nicht gleichzeitig entscheidbarer Aussagen. Dialectica 1960, 14, 239-246. [CrossRef]

17. Goldschmidt, T.; Pearce, K.L. Idealism: New Essays in Metaphysics; Oxford University Press: Oxford, UK, 2017. [CrossRef]

18. Bridgman, P.W. A Physicist's Second Reaction to Mengenlehre. Scr. Math. 1934, 2, 101-117, $224-234$.

19. Kochen, S.; Specker, E.P. The Problem of Hidden Variables in Quantum Mechanics. J. Math. Mech. 1967, 17, 59-87. [CrossRef]

20. Svozil, K. Logical equivalence between generalized urn models and finite automata. Int. J. Theor. Phys. 2005, 44, 745-754. [CrossRef]

21. Moore, E.F. Gedanken-Experiments on Sequential Machines. In Automata Studies (AM-34); Shannon, C.E., McCarthy, J., Eds.; Princeton University Press: Princeton, NJ, USA, 1956; pp. 129-153. 
22. Wright, R. The state of the pentagon. A nonclassical example. In Mathematical Foundations of Quantum Theory; Marlow, A.R., Ed.; Academic Press: New York, NY, USA, 1978; pp. 255-274.

23. Wright, R. Generalized urn models. Found. Phys. 1990, 20, 881-903. [CrossRef]

24. Gleason, A.M. Measures on the closed subspaces of a Hilbert space. J. Math. Mech. 1957, 6, 885-893. [CrossRef]

25. Godsil, C.D.; Newman, M.W. Coloring an Orthogonality Graph. SIAM J. Discret. Math. 2008, 22, 683-692. [CrossRef]

26. Halmos, P.R. Finite-Dimensional Vector Spaces; Undergraduate Texts in Mathematics; Springer: New York, NY, USA, 1958.

27. Lovász, L.; Saks, M.; Schrijver, A. Orthogonal representations and connectivity of graphs. Linear Algebra Its Appl. 1989, 114, 439-454. [CrossRef]

28. Solís-Encina, A.; Portillo, J.R. Orthogonal Representation of Graphs. arXiv 2015, arXiv:1504.03662.

29. Greechie, R.J. Orthomodular lattices admitting no states. J. Comb. Theory Ser. A 1971, 10, 119-132. [CrossRef]

30. Navara, M.; Rogalewicz, V. The pasting constructions for orthomodular posets. Math. Nachr. 1991, 154, 157-168. [CrossRef]

31. Reck, M.; Zeilinger, A.; Bernstein, H.J.; Bertani, P. Experimental realization of any discrete unitary operator. Phys. Rev. Lett. 1994, 73, 58-61. [CrossRef]

32. Cabello, A. Experimentally Testable State-Independent Quantum Contextuality. Phys. Rev. Lett. 2008, 101, 210401. [CrossRef]

33. Pitowsky, I. Infinite and finite Gleason's theorems and the logic of indeterminacy. J. Math. Phys. 1998, 39, 218-228. [CrossRef]

34. Abbott, A.A.; Calude, C.S.; Svozil, K. Value-indefinite observables are almost everywhere. Phys. Rev. A 2014, 89, 032109. [CrossRef]

35. Svozil, K.; Tkadlec, J. Greechie diagrams, nonexistence of measures in quantum logics and Kochen-Specker type constructions. J. Math. Phys. 1996, 37, 5380-5401. [CrossRef]

36. Tkadlec, J. Greechie diagrams of small quantum logics with small state spaces. Int. J. Theor. Phys. 1998, 37, 203-209. [CrossRef]

37. Boole, G. On the Theory of Probabilities. Philos. Trans. R. Soc. Lond. 1862, 152, 225-252. [CrossRef]

38. Froissart, M. Constructive generalization of Bell's inequalities. Il Nuovo Cim. B (1971-1996) 1981, 64, $241-251$. 10.1007/BF02903286. [CrossRef]

39. Tsirelson, B.S. Some results and problems on quantum Bell-type inequalities. Hadron. J. Suppl. 1993, 8, 329-345.

40. Pitowsky, I. The range of quantum probabilities. J. Math. Phys. 1986, 27, 1556-1565. [CrossRef]

41. Pitowsky, I. Quantum Probability-Quantum Logic. In Lecture Notes in Physics; Springer: Berlin/Heidelberg, Germany, 1989; Volume 321.

42. Pitowsky, I. From George Boole to John Bell: The origin of Bell's inequality. In Bell's Theorem, Quantum Theory and the Conceptions of the Universe; Kafatos, M., Ed.; Fundamental Theories of Physics; Kluwer Academic Publishers, Springer: Dordrecht, The Netherlands, 1989; Volume 37, pp. 37-49. [CrossRef]

43. Pitowsky, I. Correlation polytopes their geometry and complexity. Math. Program. 1991, 50, $395-414$. [CrossRef]

44. Pitowsky, I. George Boole's ‘Conditions of Possible Experience' and the Quantum Puzzle. Br. J. Philos. Sci. 1994, 45, 95-125. [CrossRef]

45. Pitowsky, I.; Svozil, K. New optimal tests of quantum nonlocality. Phys. Rev. A 2001, 64, 014102. [CrossRef]

46. Svozil, K. How much contextuality? Nat. Comput. 2012, 11, 261-265, [CrossRef]

47. Zierler, N.; Schlessinger, M. Boolean Embeddings of Orthomodular Sets and Quantum Logic. In The Logico-Algebraic Approach to Quantum Mechanics: Volume I: Historical Evolution; Hooker, C.A., Ed.; Springer: Dordrecht, The Netherlands, 1975; pp. 247-262. [CrossRef]

48. Tutte, W.T. A Short Proof of the Factor Theorem for Finite Graphs. Can. J. Math. 1954, 6, 347-352. [CrossRef]

49. Szabó, J. Good characterizations for some degree constrained subgraphs. J. Comb. Theory Ser. B 2009, 99, 436-446. [CrossRef] 
50. Ramanathan, R.; Rosicka, M.; Horodecki, K.; Pironio, S.; Horodecki, M.; Horodecki, P. Gadget structures in proofs of the Kochen-Specker theorem. arXiv 2018, arXiv:1807.00113.

51. Svozil, K. Physical [A]Causality. Determinism, Randomness and Uncaused Events; Springer: Cham, Switzerland; Berlin/Heidelberg, Germany; New York, NY, USA, 2018. [CrossRef]

52. Svozil, K. Quantum Scholasticism: On Quantum Contexts, Counterfactuals, and the Absurdities of Quantum Omniscience. Inf. Sci. 2009, 179, 535-541. [CrossRef]

53. Cohen, D.W. An Introduction to Hilbert Space and Quantum Logic; Problem Books in Mathematics; Springer: New York, NY, USA, 1989. [CrossRef]

54. Stairs, A. Quantum logic, realism, and value definiteness. Philos. Sci. 1983, 50, 578-602. [CrossRef]

55. Yu, S.; Oh, C.H. State-Independent Proof of Kochen-Specker Theorem with 13 Rays. Phys. Rev. Lett. 2012, 108, 030402, [CrossRef]

56. Clifton, R.K. Getting contextual and nonlocal elements-of-reality the easy way. Am. J. Phys. 1993, 61, $443-447$. [CrossRef]

57. Belinfante, F.J. A Survey of Hidden-Variables Theories. In International Series of Monographs in Natural Philosophy; Pergamon Press: Oxford, UK; Elsevier: New York, NY, USA, 1973; Volume 55. [CrossRef]

58. Pitowsky, I. Substitution and Truth in Quantum Logic. Philos. Sci. 1982, 49, 380-401. [CrossRef]

59. Hardy, L. Quantum mechanics, local realistic theories, and Lorentz-invariant realistic theories. Phys. Rev. Lett. 1992, 68, 2981-2984. [CrossRef] [PubMed]

60. Hardy, L. Nonlocality for two particles without inequalities for almost all entangled states. Phys. Rev. Lett. 1993, 71, 1665-1668. [CrossRef] [PubMed]

61. Boschi, D.; Branca, S.; De Martini, F.; Hardy, L. Ladder Proof of Nonlocality without Inequalities: Theoretical and Experimental Results. Phys. Rev. Lett. 1997, 79, 2755-2758. [CrossRef]

62. Cabello, A.; García-Alcaine, G. A hidden-variables versus quantum mechanics experiment. J. Phys. A Math. Gen. Phys. 1995, 28, 3719-3724. [CrossRef]

63. Cabello, A.; Estebaranz, J.M.; García-Alcaine, G. Bell-Kochen-Specker theorem: A proof with 18 vectors. Phys. Lett. A 1996, 212, 183-187. [CrossRef]

64. Cabello, A. No-hidden-variables proof for two spin- particles preselected and postselected in unentangled states. Phys. Rev. A 1997, 55, 4109-4111. [CrossRef]

65. Badziąg, P.; Bengtsson, I.; Cabello, A.; Granström, H.; Larsson, J.A. Pentagrams and Paradoxes. Found. Phys. 2011, 41, 414-423. [CrossRef]

66. Chen, J.L.; Cabello, A.; Xu, Z.P.; Su, H.Y.; Wu, C.; Kwek, L.C. Hardy's paradox for high-dimensional systems. Phys. Rev. A 2013, 88, 062116. [CrossRef]

67. Cabello, A.; Badziag, P.; Terra Cunha, M.; Bourennane, M. Simple Hardy-Like Proof of Quantum Contextuality. Phys. Rev. Lett. 2013, 111, 180404. [CrossRef]

68. Dvurečenskij, A.; Pulmannová, S.; Svozil, K. Partition Logics, Orthoalgebras and Automata. Helv. Phys. Acta 1995, 68, 407-428.

69. Cabello, A. A simple proof of the Kochen-Specker theorem. Eur. J. Phys. 1994, 15, 179-183. [CrossRef]

70. Cabello, A. Pruebas Algebraicas de Imposibilidad de Variables Ocultas en Mecánica Cuántica. Ph.D. Thesis, Universidad Complutense de Madrid, Madrid, Spain, 1996.

71. Svozil, K. New Forms of Quantum Value Indefiniteness Suggest That Incompatible Views on Contexts Are Epistemic. Entropy 2018, 20, 406. [CrossRef]

72. Johansen, H.B. Comment on "Getting contextual and nonlocal elements-of-reality the easy way". Am. J. Phys. 1994, 62, 471. [CrossRef]

73. Vermaas, P.E. Comment on "Getting contextual and nonlocal elements-of-reality the easy way". Am. J. Phys. 1994, 62, 658. [CrossRef]

74. Svozil, K. Physical (A)Causality. In Fundamental Theories of Physics; Springer International Publishing: Cham, Switzerland; Heidelberg, Germany; New York, NY, USA; Dordrecht, The Netherlands; London, UK, 2018; Volume 192. [CrossRef]

75. Portillo, J.R. Logical equivalence of nonseparability and "true if and only if" (TIFFTS). Universidad de Sevilla, Seville, Spain, 2018, Private Conversation.

76. Clark, K.J. Is God a Bowler or a Curler? Presented at the Randomness and Providence Workshop, Kalamazoo, MI, USA, 9 May 2017. 
77. Clavadetscher-Seeberger, E. Eine Partielle Prädikatenlogik. Ph.D. Thesis, ETH-Zürich, Zürich, Switzerland, 1983.

78. Peres, A. Unperformed experiments have no results. Am. J. Phys. 1978, 46, 745-747. [CrossRef] 